\title{
Diagnostic performance of focused cardiac ultrasound performed by emergency physicians for the assessment of ascending aorta dilatation and aneurysm
}

\author{
P Nazerian", C Gigli, A Pavellini, FR Ermini, G Pepe, S Vanni, S Grifoni \\ From 10th WINFOCUS World Congress on Ultrasound in Emergency and Critical Care \\ Kuala Lumpur, Malaysia. 16-19 November 2014
}

\section{Background}

The diagnostic performance of transthoracic focus cardiac ultrasound (FoCUS) performed by emergency physician (EP) to estimate ascending aorta dimensions in the acute setting is not known.

\section{Objective}

We investigate the accuracy and the intervariability of EP-performed FoCUS to estimate thoracic aortic dilation and aneurysm compared with computed tomography angiography (CTA) as the reference standard.

\section{Patients and methods}

This was a prospective single-centre cohort study of a convenience sample of patients that underwent CTA in the emergency department for suspected aortic pathology. FOCUS was performed before CTA and the maximum ascending aorta diameter measured in parasternal longaxis view. Diagnostic accuracy of FOCUS for detection of aortic dilation (diameter $\geq 40 \mathrm{~mm}$ ) and aneurysm (diameter $\geq 45 \mathrm{~mm}$ ) were calculated considering CTA as reference standard. In a subgroup of patients, a second EP-sonographer performed FoCUS to evaluate interobserver agreement for the diagnosis of ascending aorta dilation.

\section{Results}

140 patients were enrolled in the study. Ascending aorta dilation and aneurysm were detected at FoCUS in $50(35.7 \%)$ and in $27(17.8 \%)$ respectively. Sensitivity and specificity of FOCUS for ascending aorta dilation were 78.6\% (95\% CI 65.6-88.4) and 93.9\% (95\% CI 85.1-97.3) respectively and for ascending aorta aneurysm were $64.7 \%$ (95\% CI 46.5-80.2) and 95.3\% (95\% CI 89.3-98.4) respectively. Inter-observer agreement of FoCUS was $\mathrm{k}=0.82$.

\section{Conclusions}

FoCUS performed by EP showed a good specificity and suboptimal sensitivity for ascending aorta dilation and aneurysm when compared to CTA and appears as a highly reproducible technique.

Published: 9 March 2015

doi:10.1186/2036-7902-7-S1-A12

Cite this article as: Nazerian et al:: Diagnostic performance of focused cardiac ultrasound performed by emergency physicians for the assessment of ascending aorta dilatation and aneurysm. Critical Ultrasound Journal 2015 7(Suppl 1):A12.

\footnotetext{
* Correspondence: pnazerian@hotmail.com

Emergency Department, Careggi University Hospital, Italy
}

(c) 2015 Nazerian et al; licensee Springer. This is an Open Access article distributed under the terms of the Creative Commons Attribution License (http://creativecommons.org/licenses/by/4.0), which permits unrestricted use, distribution, and reproduction in any medium, provided the original work is properly cited. 